\title{
Contenido de glicosaminoglicanos, aldehídos y proteínas en el líquido sinovial de la articulación metacarpofalángica equina normal y alterada"
}

\author{
Concentration of glycosaminoglycan, aldehydes and protein in synovial fluid from normal \\ and damaged equine metacarpophalangeal joints
}

\author{
H Adarmes ${ }^{1 *}$, A Croxatto ${ }^{1}$, M Galleguillos ${ }^{1}$, E González ${ }^{1}$ \\ Facultad de Ciencias Veterinarias y Pecuarias, Universidad de Chile, \\ Santa Rosa 11735 La Pintana, Santiago, Chile.
}

\begin{abstract}
SUMMARY
The purpose of this study was to evaluate the influence of severity of osteoarthritis on the concentration of degradation products from the cartilage extracellular matrix on synovial fluid. Total protein and aldehyde group concentrations were determined as indicators of collagen degradation. The concentration of glycosaminoglycans (GAGs) in sinovial liquid was also determined, both the total amount (GAGsT) and the fractions corresponding to the GAGs other than hyaluronic acid or sulfated GAGs (GAGsG), that consist mostly of chondroitin and keratin sulfate. The hyaluronic acid content of the synovial fluid was calculated as the difference between the concentrations of GAGsT and GAGsS. Samples of synovial fluid were collected from metacarpophalangeal joints of crossbred equines immediatelly after slaughter, by aseptic needle aspiration and then, following post mortem joint examination, were divided into four groups: a normal group as control $(\mathrm{n}=17)$ and three alterated groups, obtained from joints with different degrees damage $(+=15 ;++=12$ and $+++=18$ samples). The synovial fluid aldehyde concentrations were determined by a colorimetric reaction between aliphatic aldehydes and N-Methyl Benzothiazolidon Hydrazone. A colorimetric method with Alcian Blue using different $\mathrm{MgCl}_{2}$ concentrations, was used to quantify GAGsT and GAGsS. These results showed an increase of total protein concentration in the more alterated group $(+++)(\mathrm{P}<0,05)$. The collagen degradation product concentration increased in the group less altered $(+)$ compared whith the more alterated group $(+++)$ and collagen protein concentration showed an increase in both less alterated groups $(+$ and ++$)$ compared with the more alterated group $(+++)(\mathrm{P}<0,05)$. The GAGsT, GAGsS and hyaluronic acid concentrations did not show statisticant differences between groups. GAGsT and hyaluronic acid concentrations showed a tendency to decrease in the more damaged samples. These results indicate that collagen degradation product concentration in synovial fluid could be used as a marker of initial changes in the osteoarthritis process.
\end{abstract}

Palabras clave: líquido sinovial, colágeno, glicosaminoglicanos.

Key words: synovial fluid, collagen, glycosaminoglycans.

\section{INTRODUCCION}

La articulación metacarpofalángica del equino cursa frecuentemente con procesos de naturaleza inflamatoria y degenerativa (Maldonado y col 1983). El cartílago articular de las articulaciones móviles está formado por condrocitos, incluidos en una abundante matriz extracelular (MEC) de colágeno tipo II y de proteoglicanos con sus glicosaminoglicanos (GAGs) (Todhunter, 1996). Los condrocitos se encargan de regular activamente la producción y destrucción de estos componentes de la MEC del cartílago (Morris y Treadwell 1994).

Aceptado: 14.06.2005.

\# Proyecto DTI - A - 3135/1992. Universidad de Chile.

* Fax 6785526, hadarmes@uchile.cl, casilla 2 correo 15, Santiago.
El principal colágeno del cartílago articular es el fibrilar tipo II, compuesto por tres cadenas polipeptídicas $\alpha 1$ (II), cuyos extremos corresponden a zonas extendidas no helicoidales, mientras la región comprendida entre ellas, que representa aproximadamente el 95\% de la molécula, se organiza formando una triple hélice estabilizada por entrelazos intermoleculares, en los que participan las formas aldehídicas alisina e hidroxialisina que proporcionan resistencia a las fuerzas de tracción (Ricard-Blum y Ville 1989).

Los proteoglicanos, junto al colágeno tipo II, constituyen los componentes orgánicos cuantitativamente más importantes de la MEC del cartílago y son responsables de gran parte de las propiedades fisicoquímicas del tejido (Bayliss y col 1999, Räsänen y Messner 1999). Los proteoglicanos están formados por una proteína central, a la que se unen covalentemente los GAGs, siendo el agrecán el proteoglicano más abundante (aproximadamente $90 \%)$ y el de mayor tamaño $\left(3 \times 10^{6} \mathrm{Da}\right)$ (Palmer 
y Bertone 1994, Hedlund y col 1999). La capacidad de amortiguación del cartílago se debe a la gran retención agua y a la repulsión de los grupos aniónicos de los proteoglicanos (Palmer y Bertone 1994, McIlwraith 1996, Räsänen y Messner 1999).

El agrecán forma grandes estructuras macromoleculares en la MEC del cartílago articular, a través de su unión no covalente con una molécula de ácido hialurónico (Tulamo y col 1996, Maroudas y col 1998). La formación de estos agregados es muy importante desde el punto de vista fisiológico, ya que permite la retención del agrecán y por ende de agua en la MEC del cartílago, asegurándose así la amortiguación normal del cartílago articular (Maroudas y col 1998).

El recambio de la MEC del cartílago está regulado por una serie de factores relacionados con la distribución de la carga mecánica y la presencia de algunas moléculas como el factor de crecimiento tipo insulina (IGF) I y II y el factor de crecimiento transformante $\beta$, que estimularían la síntesis de MEC. Por otro lado, la interleuquina 1 (IL-1) (Morris y Treadwell 1994) y el factor de necrosis tumoral $\alpha$ (Billinghurst y col 1995) producidos por sinoviocitos y condrocitos inhibirían la síntesis de matriz extracelular e inducirían la síntesis y activación de enzimas denominadas metaloproteinasas (MMPs) que degradan los componentes de la matriz extracelular del cartílago (Yoshihara y col 2000; Jin y col 2003). Los colágenos fibrilares presentan una rigidez y gran resistencia a la hidrólisis proteolítica, relacionada con sus entrelazos intermoleculares, que determinan que su recambio molecular sea lento. Se ha descrito que la colagenasa tisular o intersticial o MMP-1 separa las tres cadenas $\alpha 1$ (II) de la triple hélice en puntos específicos del colágeno tipo II (McIlwraith 1996).

Los proteoglicanos poseen un recambio más dinámico y la hidrólisis de la proteína central en sitios específicos por acción de las MMPs provoca la liberación de grandes fragmentos de proteoglicanos que difunden fuera del tejido hacia la cavidad sinovial (Todhunter 1996).

Las MMPs son a su vez inhibidas por dos inhibidores tisulares de las MMPs (TIMPs): los TIMP - 1 y TIMP 2 (McIlwraith 1996). El desbalance del recambio de la matriz provocado por la pérdida de regulación de la síntesis y de la actividad de las MMPs provoca la pérdida gradual de componentes de la MEC del cartílago que conduce finalmente al desarrollo de la enfermedad degenerativa articular $u$ osteoartritis, que se describe como progresiva, estructural y funcional, con deterioro del cartílago articular y acompañada de un intento de reparación de las superficies articulares con neoformación ósea (McIlwraith 1996).

Existen trabajos cuyo objetivo ha sido caracterizar la pérdida de componentes de la matriz durante la enfermedad degenerativa articular, a través de relacionar la concentración de colágeno (Maldonado y col 1983), GAGs totales y de queratán sulfato del líquido sinovial con el daño del cartílago en las patologías articulares (Todhunter y col 1997, Okumura y Fujinaga 1998). Además, se ha descrito que la concentración de queratán sulfato representaría mejor a los componentes propios de la matriz extracelular del cartílago (Todhunter y col 1997). El daño del colágeno del cartílago articular constituiría un evento central en la patogénesis del envejecimiento del cartílago y de la osteoartritis (Tiku y col 2003).

En este trabajo se planteó como hipótesis la relación directa entre la severidad anatómica del cuadro osteoartrítico y la concentración de componentes de la MEC del cartílago en el líquido sinovial. Para esto se evaluó la concentración total de proteínas, de GAGs distintos del ácido hialurónico y de grupos aldehídicos. Además, se estimó la concentración de ácido hialurónico del líquido sinovial.

\section{MATERIAL Y METODOS}

Se utilizó líquido sinovial obtenido por artrocéntesis (Byars y col 1982), desde la articulación metacarpofalángica de equinos mestizos mayores de tres años, según cronometría dentaria y recién beneficiados en el matadero "La Pintana" (Región Metropolitana). Se clasificaron 17 muestras como normales y 45 como patológicas, de acuerdo al aspecto macroscópico de la articulación. Se clasificaron como normales aquellos líquidos sinoviales obtenidos de articulaciones cuyo cartílago era de color blanco nacarado, con su superficie lisa y brillante, sin líneas de roce o zonas de erosión. Además, la membrana sinovial no debía presentar signos de congestión, mientras que el líquido sinovial debía ser de color amarillo claro, transparente, sin flóculos ni trazas de material sanguinolento (González y col 1994, Adarmes y col 2003).

A su vez, las muestras patológicas se dividieron en tres grupos $(+,++,+++)$ según la magnitud de las lesiones. Así, una cruz $(+)(\mathrm{n}=15)$ correspondió a aquellas articulaciones cuyos cartílagos presentaban cambios leves de color con líneas de roce. Dos cruces $(++)(n=12)$ correspondió a francos cambios de coloración del cartílago articular, con líneas de roce manifiestas y pequeños focos de erosión. Tres cruces $(+++)(n=18)$ correspondió a alteraciones del cartílago en su grado máximo, con grandes focos de erosión (González y col 1994). El líquido sinovial obtenido se mantuvo en hielo y luego se realizó su procesamiento en el laboratorio, para lo cual se centrifugó a $950 \mathrm{x}$ g durante 20 minutos a $4{ }^{\circ} \mathrm{C}$. Los sobrenadantes se congelaron a $-20{ }^{\circ} \mathrm{C}$, para realizar luego las siguientes determinaciones, previa dilución 1:4 de estas muestras de líquido sinovial.

En la determinación de proteínas (Lowry y col 1951) se utilizó seroalbúmina de bovino al $0,1 \%$, a partir de la cual se preparó una curva estándar con un rango de cantidad de proteína entre 50 y $500 \mu \mathrm{g}$, estableciéndose la absorbancia espectrofotométricamente a $595 \mathrm{~nm}$. Los re- 
sultados se expresaron como $g$ de proteína/L de líquido sinovial.

Para la determinación de grupos aldehídos asociados al colágeno, se utilizó el método descrito por Sawiki y col (1961), modificado por Paz y col (1965) y adaptado por Horwath y Tabora (1972). A partir de una solución de acetaldehído $10^{-3} \mathrm{M}$ se preparó una curva estándar en un rango de cantidad de acetaldehído entre 2,0 y $8,8 \mu \mathrm{g}$. La absorbancia se determinó espectrofotométricamente a $670 \mathrm{~nm}$, expresándose los resultados como g de aldehídos/L de líquido sinovial. Esta técnica consiste en la formación de un complejo coloreado, por la reacción de los aldehídos alifáticos con el N-Metil Benzotiazolidon Hidrazona (MBTH) al $1 \%$ en presencia de $\mathrm{FeCl}_{3}$ al $0,2 \%$. Para esto se utilizaron $0,8 \mathrm{ml}$ de una solución diluida (1:4) de líquido sinovial y $0,2 \mathrm{ml}$ de MBTH al 1\%. Después de 30 minutos a temperatura ambiente se agregaron 2,5 $\mathrm{ml} \mathrm{de} \mathrm{FeCl}_{3}$ al 0,2\% y 6,5 ml de acetona. Para determinar la concentración de GAGs totales (GAGs T) y de GAGs diferentes del ácido hialurónico (GAGs S) se utilizó el método colorimétrico de Bartold y Page (1985) descrito por Adarmes y col (2003).

Finalmente se realizó la estimación del contenido de ácido hialurónico al calcular la diferencia entre GAGs T y GAGs S.

Análisis estadístico. Mediante la prueba de KolmogorovSmirnov se estableció que los datos experimentales no difieren significativamente de una distribución normal. Los valores experimentales de cada grupo se sometieron a un análisis de varianza y luego se realizó la prueba de Duncan con el fin de establecer las diferencias entre grupos homogéneos (Zar 1996) utilizando un programa Computacional Statistica 6.0. Se consideró un nivel de significancia de $\mathrm{P}<0,05$. Los valores obtenidos se expresan como media \pm DE para cada variable analizada.

\section{RESULTADOS}

La concentración de proteínas totales del líquido sinovial refleja los procesos de ultrafiltración del plasma sanguíneo en la membrana sinovial y el recambio molecular de las estructuras articulares. Los resultados indican que la concentración de proteínas fue significativamente mayor $(\mathrm{P}<0.05)$ sólo en el grupo con daño más severo $(+++)$ (cuadro 1$)$.

La concentración de residuos aldehídicos en el líquido sinovial (cuadro 2) que representan los productos de degradación del colágeno mostró un incremento significativo en el grupo menos alterado $(+)$ con respecto al más alterado $(+++)(\mathrm{P}<0,05)$. Con la finalidad de establecer la proporción de proteína colagenosa en el líquido sinovial, se calculó la relación aldehídos/proteínas, encontrándose que ésta disminuyó en forma significativa $(\mathrm{P}<0,05)$ en el grupo más alterado $(+++)$ con respecto a los grupos menos alterados $(+\mathrm{y}++)$.
La concentración de GAGs T y de GAGs S en el líquido sinovial no mostró variaciones significativas entre los grupos analizados $(\mathrm{P}>0,05)$, observándose en el caso de los GAGs $\mathrm{T}$ una tendencia a disminuir en el grupo más alterado $(+++)$ comparado con el normal, mientras que los GAGs $\mathrm{S}$ muestran una tendencia a aumentar en el grupo más alterado $(+++)$ (cuadro 3). Con respecto al contenido de ácido hialurónico del líquido sinovial, tampoco se encontraron variaciones significativas entre los grupos analizados $(\mathrm{P}>0,05)$, observándose sólo una tendencia a disminuir $(\mathrm{P}<0,08)$ en el grupo con mayor daño articular comparada con el grupo normal.

Cuadro 1. Concentración promedio $\pm \mathrm{DE}$ de proteínas $(\mathrm{g} / \mathrm{L})$ en el líquido sinovial de la articulación metacarpofalángica equina.

Average $( \pm \mathrm{SD})$ synovial fluid protein concentration $(\mathrm{g} / \mathrm{L})$ in equine metacarpophalangeal joints

\begin{tabular}{lc}
\hline Clasificación de la articulación & Proteínas $(\mathrm{g} / \mathrm{L})$ \\
\hline Normal $(\mathrm{n}=17)$ & $11,7 \pm 2,4^{\mathrm{a}}$ \\
Alterada $+(\mathrm{n}=15)$ & $12,4 \pm 2,8^{\mathrm{a}}$ \\
Alterada $++(\mathrm{n}=12)$ & $12,1 \pm 4,3^{\mathrm{a}}$ \\
Alterada $+++(\mathrm{n}=18)$ & $15,7 \pm 6,3^{\mathrm{b}}$ \\
\hline
\end{tabular}

Letras diferentes indican diferencias estadísticamente significativas.

Cuadro 2. Concentración promedio \pm DE de aldehídos $(\mathrm{g} / \mathrm{L})$ y relación $\mathrm{g}$ aldehídos/g proteínas $\pm \mathrm{DE}$ en el líquido sinovial de la articulación metacarpofalángica equina.

Average $( \pm$ SD) aldehyde concentration and relationship between aldehydes and protein concentration in equine metacarpophalangeal joints.

\begin{tabular}{lcc}
\hline $\begin{array}{c}\text { Clasificación de } \\
\text { la articulación }\end{array}$ & $\begin{array}{c}\text { Aldehídos } \\
(\mathrm{g} / \mathrm{L}) \times 10^{-3}\end{array}$ & $\begin{array}{c}\text { Relación } \\
\text { aldehídos/ proteínas } \\
\times 10^{-3}\end{array}$ \\
\hline Normal $(\mathrm{n}=17)$ & $4,06 \pm 1,3^{\mathrm{ab}}$ & $0,35 \pm 0,1^{\mathrm{ab}}$ \\
Alterada $+(\mathrm{n}=15)$ & $5,34 \pm 2,4^{\mathrm{a}}$ & $0,45 \pm 0,2^{\mathrm{a}}$ \\
Alterada $++(\mathrm{n}=12)$ & $4,42 \pm 1,8^{\mathrm{ab}}$ & $0,41 \pm 0,2^{\mathrm{a}}$ \\
Alterada $+++(\mathrm{n}=18)$ & $3,41 \pm 1,1^{\mathrm{b}}$ & $0,25 \pm 0,1^{\mathrm{b}}$ \\
\hline
\end{tabular}

Letras diferentes indican diferencias estadísticamente significativas.

Cuadro 3. Concentración promedio de GAGs totales (GAGs T), de GAGs distintos del ácido hialurónico (GAGs $\mathrm{S}$ ) y de ácido hialurónico (A.H.) (g/L) $\pm \mathrm{DE}$ del líquido sinovial de la articulación metacarpofalángica equina.

Average ( \pm SD) concentrations of total GAGs (GaGs T), GAGs other than hyaluronic acid (GAGs S) and hyaluronic acid (A.H.) in synovial fluid from equine metacarpophalangeal joints.

\begin{tabular}{lccc}
\hline $\begin{array}{l}\text { Clasificación de } \\
\text { la articulación }\end{array}$ & $\begin{array}{c}\text { GAGs T } \\
(\mathrm{g} / \mathrm{L})\end{array}$ & $\begin{array}{c}\text { GAGs S } \\
(\mathrm{g} / \mathrm{L})\end{array}$ & $\begin{array}{l}\text { A.H. } \\
(\mathrm{g} / \mathrm{L})\end{array}$ \\
\hline Normal $(\mathrm{n}=17)$ & $1,91 \pm 0,4$ & $0,31 \pm 0,2$ & $1,57 \pm 0,5$ \\
Alterada $+(\mathrm{n}=15)$ & $1,72 \pm 0,5$ & $0,27 \pm 0,09$ & $1,44 \pm 0,4$ \\
Alterada $++(\mathrm{n}=12)$ & $1,84 \pm 0,4$ & $0,37 \pm 0,2$ & $1,47 \pm 0,5$ \\
Alterada $+++(\mathrm{n}=18)$ & $1,60 \pm 0,3$ & $0,41 \pm 0,2$ & $1,19 \pm 0,3$ \\
\hline
\end{tabular}




\section{DISCUSION}

Para la obtención de un diagnóstico precoz de la alteración del cartílago articular se ha buscado detectar y/o medir la concentración de moléculas propias de la MEC del cartílago articular, en diferentes líquidos biológicos que incluyen el fluido sinovial (Maldonado y col 1983, Thonar y col 1985; Little y col 1990; Alwan y col 1991; Fuller y col 1996; Todhunter y col 1997; Okumura y Fujinaga, 1998; Frisbie y col 1999). Se evaluó en el líquido sinovial de la articulación metacarpofalángica equina con distintos grados de alteración el contenido del conjunto de componentes orgánicos más abundantes de la MEC del cartílago, como son el colágeno y los GAGsS.

La concentración de proteínas del líquido sinovial no constituye sólo un reflejo de la degradación de la MEC, sino que también del proceso de ultrafiltración a nivel de la membrana sinovial y la razón de medir su concentración se relacionó con el alto contenido de proteína colagenosa de la MEC, que es aproximadamente dos tercios el peso seco del cartílago articular adulto (Eyre 2002) y con la finalidad de utilizar este parámetro para determinar la proteína colagenosa a través de la relación de aldehídos/proteínas totales. Esta concentración se encontró aumentada significativamente $(\mathrm{P}<0,05)$ en el grupo más alterado, lo que concuerda con lo descrito por Tew (1982). Este incremento podría reflejar una mayor degradación de los componentes de la MEC del cartílago por acción de diversas metaloproteinasas, o bien, una alteración en la capacidad de ultrafiltración de la membrana sinovial, aunque esta alteración se asocia especialmente a los procesos de naturaleza inflamatoria aguda (Tew 1980).

El desbalance entre la síntesis y degradación del colágeno, así como el incremento de la actividad de las colagenasas (Squires y col 2003), se relaciona con alteraciones del colágeno del cartílago articular, lo que constituye un evento central en la patogénesis del envejecimiento del cartílago y de la enfermedad degenerativa articular (Tiku y col 2003),

La concentración de grupos aldehídos representativos de los productos de degradación del colágeno del cartílago aumentó significativamente en el grupo menos dañado $(+)$ respecto del más dañado $(+++)$. Con el objetivo de relacionar el contenido de aldehídos con el carácter colagenoso de la proteína, se estableció la relación aldehídos/proteínas, la que también demostró una disminución significativa $(\mathrm{P}<0,05)$ del componente colagenoso del líquido sinovial, a medida que el daño se hace más severo, al compararlo con los grupos con daño leve $(+)$ y moderado $(++)$. Esto se podría asociar a la mayor degradación de colágeno en los procesos iniciales, debido a la liberación de mediadores de la inflamación desde la membrana sinovial, con activación de MMPs que afecten la superficie del cartílago y a la pre- sencia de colágeno menos entrelazado en esa zona. Por otro lado, los procesos articulares degenerativos aumentan con la edad, factor que también influye en el incremento de la cantidad de entrelazos del colágeno del tipo de la hidroxilisilpiridinolina y lisilpiridinolina (RicardBlum y Ville 1989), o bien del tipo de la pentosidina (Brama y col 1999), menos degradables por acción enzimática, lo que podría explicar la menor cantidad de proteína colagenosa en el líquido sinovial del grupo más alterado.

En este resultado se debe considerar también la metodología utilizada, por cuanto la reacción del MBTH permite el reconocimiento de grupos aldehídos, que se asocian posiblemente a productos de degradación de colágeno menos entrelazados, y por el contrario, al aumentar la madurez del colágeno con la formación de entrelazos más irreversibles, los grupos aldehídos podrían encontrarse menos disponibles para la reacción con el MBTH.

Por otro lado, los GAGs T, los GAGs S y el ácido hialurónico no mostraron cambios significativos entre los grupos analizados, detectándose una tendencia a disminuir del ácido hialurónico en el grupo más alterado $(+++)$, lo que también ha sido descrito por otros autores (Tulamo y col 1996) y que predispondría a incrementar aún más el daño mecánico del cartílago articular.

Además, la concentración de GAGs S representativos de la MEC del cartílago aumentó en forma no significativa en los tres grupos alterados, siendo esta tendencia mayor en el grupo más alterado $(+++)$, lo que podría sugerir que a medida que aumenta el daño del cartílago, se produce mayor degradación de los proteoglicanos de la MEC del cartílago por acción de metaloproteinasas del tipo de la estromelisina o MMP-3 (Brama y col 2000) o bien se produce la síntesis de moléculas de proteoglicanos que no logran formar parte de MEC del cartílago, con la pérdida consecuente de los GAGs S hacia el líquido sinovial.

Estos resultados podrían indicar la pérdida asincrónica de los componentes colágeno y GAGs de la MEC del cartílago articular en que la pérdida inicial de la red de colágeno predispondría a la degradación posterior de los proteoglicanos, lo que se puede asociar a una activación diferenciada de las cascadas enzimáticas relacionadas con el proceso degradativo.

Se puede concluir que: la concentración de proteínas del líquido sinovial de la articulación metacarpofalángica equina fue mayor en el grupo con osteoartritis severa $(+++)$. La concentración de grupos aldehído del líquido sinovial de la articulación metacarpofalángica equina, representativos de productos de degradación del colágeno, se encontró aumentada en la osteoartritis leve $(+)$, mientras que el carácter colagenoso de la proteína del líquido sinovial fue mayor en la osteoartritis leve $(+)$ y moderada (++). La concentración de GAGs T, de GAGs $\mathrm{S}$ y de ácido hialurónico no mostró cambios significati- 
vos entre los grupos analizados; sólo la concentración de ácido hialurónico mostró una tendencia a disminuir en el grupo con osteoartritis severa $(+++)$.

\section{RESUMEN}

El objetivo del trabajo fue relacionar la severidad del cuadro osteoartrítico con la concentración de moléculas de la matriz extracelular del cartílago en el líquido sinovial. Para ello se midieron las concentraciones de proteína total y de grupos aldehídos como productos de degradación del colágeno. Además, se midió la concentración de glicosaminoglicanos (GAGs) del líquido sinovial en su valor total (GAGs T) y de una fracción de éstos, que corresponde a los GAGs diferentes del ácido hialurónico o GAGs sulfatados (GAGs S), entre los que se describen esencialmente condroitín sulfato y queratán sulfato. El contenido de ácido hialurónico se calculó por diferencia entre las concentraciones de GAGs T y de GAGs S. Las muestras de líquido sinovial se obtuvieron por artrocéntesis aséptica desde articulaciones metacarpofalángicas de equinos mestizos en matadero, inmediatamente después del beneficio de los animales y luego separadas en cuatro grupos, después del examen visual post mortem de las articulaciones: uno normal como control $(\mathrm{n}=17)$ y tres grupos alterados provenientes de articulaciones con diferentes grados de alteración articular $(+=15 ;++=12 \mathrm{y}+++=18)$. La concentración de grupos aldehidos se determinó a través de una reacción colorimétrica entre aldehídos y N-Metil Benzotiazolidon Hidrazona. Además, para cuantificar los GAGs T y los GAGs S se utilizó un método colorimétrico con Alcian Blue usando diferentes concentraciones de $\mathrm{MgCl}_{2}$. El grupo con daño articular más severo $(+++)$ mostró incremento de la concentración de proteínas y disminución de productos de degradación de colágeno y de la proteína colagenosa $(\mathrm{P}<0,05)$, mientras que la concentración de GAGs T, de GAGs S y de ácido hialurónico no mostró diferencias significativas entre los grupos analizados $(\mathrm{P}>0,05)$, detectándose sólo tendencias a disminuir en la concentración ácido hialurónico y a aumentar en la concentración de GAGs S en el grupo con daño articular más severo $(+++)$. Estos resultados indican que la concentración de productos de degradación del colágeno en el líquido sinovial podría ser utilizada como marcador precoz de los cambios iniciales del proceso osteoartrítico.

\section{REFERENCIAS}

Adarmes H, A Riveros, M Galleguillos, E González. 2003. Contenido de glicosaminoglicanos del líquido sinovial de la articulación metacarpofalángica de caballos castrados y yeguas de diferentes edades. Arch Med Vet 35, 51-59.

Alwan WH, SD Carter, D Bennett, GB Edwards. 1991. Glycosaminoglycans in horses with osteoarthritis. Equine Vet $J$ 23, 44-47.

Bartold PM, RC Page. 1985. A microdetermination method for assaying glycosaminoglycans and proteoglycans. Annal Biochem 150, 320-324

Bayliss MT, D Osborne, S Woodhouse, C Davidson. 1999. Sulfation of chondroitin sulfate in human articular cartilage. The effect of age, topographical position, and zone of cartilage on tissue composition. J Biol Chem 274, 1589215900 .
Billinghurst RC, PB Fretz, JR Gordon. 1995. Induction of intraarticular tumour necrosis factor during acute inflammatory responses in equine arthritis. Equine Vet J 27, 208-216.

Brama PA, JM Tekoppele, RA Bank, PR van Weeren, A Barneveld. 1999. Influence of site and age on biochemical characteristics of the collagen network of equine articular cartilage. Am J Vet Res 60, 341-345.

Brama PA, JM Tekoppele, B Beekman, van El, A Barneveld, PR van Weeren. 2000. Influence of development and joint pathology on stromelysin enzyme activity in equine synovial fluid. Ann Rheum Dis 59, 155-157.

Byars TD, C Brown, D Beisel. 1982. Equine Arthrocentesis. Equine Pract 4, 28-32.

Eyre, D. 2002. Collagen of articular cartilage. Arthritis Res 4, $30-35$.

Frisbie DD, CS Ray, M Ionescu, AR Poole, PL Chapman, CW McIlwraith. 1999. Measurement of synovial fluid and serum concentrations of the 846 epitope of chondroitin sulfate and of carboxy propeptides of type II procollagen for diagnosis of osteochondral fragmentation in horses. Am $J$ Vet Res 60, 306-309.

Fuller CJ, AR Barr, PA Dieppe, M Sharif. 1996. Variation of an epitope of keratan sulphate and total glycosaminoglycans in normal equine joints. Equine Vet $J$ 28, 490-493.

González E, H Adarmes, F Fredes. 1994. Contribución a la caracterización del proceso degenerativo en el cartílago articular equino. Av Cs Vet 9, 43-48.

Hedlund H, E Hedbom, D Heinegard, S Mengarelli-Widholm, FP Reinholt, O Svensson. 1999. Association of the aggrecan keratan sulfate-rich region with collagen in bovine articular cartilage. J Biol Chem 274, 5777-5781.

Horvath A, E Tabora. 1972. Alterations of collagen in psoriatic skin. Dermatologica 144, 83-91.

Jin M, GR Emkey, P Siparsky, SB Trippel, AJ Grodzinsky. 2003. Combined effects of dynamic tissue shear deformation and insulin-like growth factor in chondrocyte biosynthesisin cartilage explants. Arch Biochem Biophys 414, 223-231.

Little CB, BJ Hilbert, S Wickstrom, BE Hedlund. 1990. Quantitative microanalysis of equine synovial fluid glycosaminoglycan concentration. Am J Vet Res 51, 15341539.

Lowry OH, NJ Rosebrough, AL Farr, RJ Randall. 1951. Protein measurement with the Folin's phenol reagent. J Biol Chem 193, 265-275.

Maldonado R, H Garcés, J Auba, A Horvath. 1983. Synovial aldehyde groups in equine joint disease. Equine Vet $J 15$, 168-169.

Maroudas A, MT Bayliss, N Uchitel-Kaushansky, R Schneiderman, E Gilav. 1998. Aggrecan turnover in human articular cartilage: use of aspartic acid racemization as a marker of molecular age. Arch Biochem Biophys 350, 61-71.

McIlwraith CW. 1996. General pathobiology of the joint and response to injure. En Mcllwraight, C.W., G.W. Trotter. Joint disease in the horse. W.B. Saunders. Philadelphia, USA.

Morris EA, BV Treadwell. 1994. Effect of interleukin 1 on articular cartilage from young and aged horses and comparison with metabolism of osteoarthritic cartilage. Am $J$ Vet Res 55, 138-146. 
Okumura M, T Fujinaga. 1998. Establisment of monoclonal antibody $(1 / 14 / 16 \mathrm{H} 9)$ for detection of equine keratan sulfate. Am J Vet Res 59, 1203-1208.

Palmer JL, AL Bertone. 1994. Joint structure, biochemistry and biochemical disequilibrium in sinovitis and equine joint disease. Equine Vet J 26, 263-277.

Paz MA, OO Blumenfeld, M Rojkind, E Henson, C Furfine, PM Gallop. 1965. Determination of carbonyl compounds with 3-methyl-2-benzothiazolone hydrazone. Arch Biochem Biophys 109, 548-559.

Räsänen T, K Messner.1999. Articular cartilage compressive stiffness following oophorectomy or treatment with 17 $\alpha$-estradiol in young postpubertal rabbits. Acta Obstet Gynecol Scand 78, 357-362.

Ricard-Blum S, G Ville. 1989. Collagen cross-linking. Int J Biochem 21(11), 1185-1189.

Sawiki E, TR Hauser, TW Stanley, W Elbert. 1961. The 3-methyl-2-benzothiazolone hydrazone test. Sensitive new methods for the detection, rapid estimation, and determination of aliphatic aldehydes. Annal Chem 33(1), 93-96.

Squires GR, S Okouneff, M Ionescu, AR Poole. 2003. The pathobiology of focal lesion development in aging human articular cartilage and molecular matrix changes characteristic of osteoarthritis. Arthritis Rheum 48, 12611270.

Tew WP. 1980. Synovial fluid particle analysis in equine joint disease. Med Vet Pract 61, 993-997.
Tew WP. 1982. Synovial fluid analysis: application in equine joint injury and disease. Proc. Annual Convention Amer. Assoc. Equine Pract 28, 121-127.

Thonar EJ-MA, ME Lenz, GK Klintworth. 1985. Quantification of keratan sulfate in blood as a marker of cartilage catabolism. Arthritis Rheum 28, 1367-1376.

Tiku ML, GT Allison, K Naik, SK Karry. 2003. Malondialdehyde oxidation of cartilage collagen by chondrocytes. Osteoarthritis Cartilage 11(3), 159-166.

Todhunter RJ. 1996. Anatomy and physiology of synovial joints. En: McIlwraith CW, Trotter GW. Joint disease in the horse.W.B. Saunders, Philadelphia, USA.

Todhunter RJ, SL Fubini, KP Freeman, G Lust. 1997. Concentrations of keratan sulfate in plasma and synovial fluid from clinically normal horses and horses with joint disease. J Am Vet Med Assoc 210, 369-374.

Tulamo R, J Houttu, A Tupamäki, M Salonen. 1996. Hyaluronate and large molecular weight proteoglycans in synovial fluid from horses with vaious arthritides. Am J Vet Res 57, 932-937.

Yoshihara Y, H Nakamura, K Obata, H Yamada, T Hayakawa, K Fujikawa, Y Okada. 2000. Matrix metalloproteinases and tissue inhibitors of metalloproteinases in synovial fluid from patients with rheumatoid arthritis or osteo-arthritis. Ann Rheum Dis 59, 455-461.

Zar JH. 1996. Biostatistical Analysis. $3^{\text {rd }}$ ed. Prentice-Hall Englewood Cliff. New Jersey, USA. 